\title{
The Therapeutic Use of Analgesics in Patients With Liver Cirrhosis: A Literature Review and Evidence-Based Recommendations
}

\author{
Farnad Imani ${ }^{1}$; Mahsa Motavaf ${ }^{2,3}$; Saeid Safari ${ }^{1}$; Seyed Moayed Alavian ${ }^{3,4,{ }^{*}}$ \\ ${ }_{1}^{1}$ Department of Anesthesiology and Pain Medicine, Iran University of Medical Sciences, Tehran, IR Iran \\ 2 Department of Genetics, Faculty of Biological Sciences, Tarbiat Modares University, Tehran, IR Iran \\ 3 Department of Molecular Hepatology, Middle East Liver Disease Center, Tehran, IR Iran \\ 4 Department or Molecular Hepatology, Middle East Liver DiseaseCenter, Tehran, IR lran \\ ${ }^{*}$ Corresponding Author: Seyed Moayed Alavian, Department of Molecular Hepatology, Middle East Liver Disease Center, Tehran, IR Iran. Tel: +98-2188945186, Fax: +98-2188945188, \\ E-mail:alavian@thc.ir
}

Received: September 11, 2014; Revised: September 30, 2014; Accepted: October 4, 2014

\begin{abstract}
Context:Pain management in cirrhotic patients is a major clinical challenge for medical professionals. Unfortunately there are no concrete guidelines available regarding the administration of analgesics in patients with liver cirrhosis. In this review we aimed to summarize the available literature and suggest appropriate evidence-based recommendations regarding to administration of these drugs.

Evidence Acquisition: An indexed MEDLINE search was conducted in July 2014, using keywords "analgesics", "hepatic impairment", "cirrhosis", "acetaminophen or paracetamol", "NSAIDs or nonsteroidal anti-inflammatory drugs", "opioid" for the period of 2004 to 2014. All randomized clinical trials, case series, case report and meta-analysis studies with the above mentioned contents were included in review process. In addition, unpublished information from the Food and Drug Administration are included as well.

Results: Paracetamol is safe in patients with chronic liver disease but a reduced dose of 2-3 g/d is recommended for long-term use. Non-steroidal anti-inflammatory drugs (NSAIDs) are best avoided because of risk of renal impairment, hepatorenal syndrome, and gastrointestinal hemorrhage. Most opioids can have deleterious effects in patients with cirrhosis. They have an increased risk of toxicity and hepatic encephalopathy. They should be administrated with lower and less frequent dosing in these patients and be avoided in patients with a history of encephalopathy or addiction to any substance.

Conclusions: No evidence-based guidelines exist on the use of analgesics in patients with liver disease and cirrhosis. As a result pain management in these patients generates considerable misconception among health care professionals, leading under-treatment of pain in this population. Providing concrete guidelines toward the administration of these agents will lead to more efficient and safer pain management in this setting.
\end{abstract}

Keywords: Adverse Drug Events; Hepatic Cirrhosis; Pain Management; Acetaminophen

\section{Context}

Liver cirrhosis is a major public health problem, accounting for approximately 770,000 deaths annually. Based on autopsy studies, its prevalence is $4.5 \%$ to $9.5 \%$ in the general population, which means that hundreds of millions of people are affected worldwide $(1,2)$. The most common causes of cirrhosis are long-term alcohol abuse, hepatitis B and C infection, autoimmune hepatitis and fatty liver disease $(3,4)$. The liver has a major role in the metabolism and pharmacokinetics of most drugs. Thus, the use of medications in patients with cirrhosis frequently raises several concerns, especially for gastroenterologists and hepatologists who are often asked for their opinion regarding the safety of drugs in this setting. One of the difficult clinical challenges for healthcare professionals is the management of pain in the patient with cirrhosis.

Despite the fact that pain relief is essential for improving the quality of life of every patient, healthcare providers often consider the use of analgesics in patients with cirrhosis as unsafe, leading to under treatment of pain in this population $(5,6)$. The most important and concerning complications in prescribing analgesics to cirrhotic patients stem from the risk of precipitating or worsening renal failure, provoking hepatic encephalopathy, inducing portal hypertension and gastrointestinal bleeding. Furthermore, because of the altered drug metabolism and pharmacokinetics in these patients, there is also an increased risk for over-sedation, under-sedation and constipation with opioids.

Even though, adverse events from analgesics are frequently possible, potentially fatal, and often preventable, guidelines for the use of these compounds in this setting are not readily available and there is a relative lack of research in this area. Thus the aim of this review was to resume and analyze the published data on various analgesics in patients with liver cirrhosis to provide possible evidence based guidelines for the safe use of these drugs in this population. 


\section{Evidence Acquisition}

A review of the available literature on the safety of analgesics administration in cirrhotic patients was conducted in July 2014. Electronic search was performed from several databases including: PubMed, Google Scholar, Scopus, Cochrane Database for the key words: "analgesics", "hepatic impairment”, “cirrhosis", "acetaminophen or paracetamol", "NSAIDs or nonsteroidal anti-inflammatory drugs", "opioid" for the period of 2004 to 2014. All randomized clinical trials, case series, case reports and meta-analysis studies with the above mentioned contents were included in review process. Unpublished information from the Food and Drug Administration (FDA) are included as well.

\section{Results}

\subsection{Hepatic Drug Metabolism in Cirrhotic Liver}

Liver plays a major role in the distribution and elimination of most drugs, including analgesic compounds. Generally, drug metabolism in the liver occurs via 3 mechanisms:1) Oxidation, reduction, or hydrolysis reactions via hepatic cytochrome P450 (CYP) enzymes, 2) Conjugation to glucuronic acid, sulfate, acetate, glycine, glutathione, or a methyl group, 3) Biliary excretion and elimination (79). The efficiency of drug removal by the liver, so-called hepatic clearance relies on hepatic blood flow, plasma protein binding and the metabolic activity of hepatic enzymes (known as intrinsic clearance).

Cirrhosis affects all of these processes and alters the metabolism of many drugs through a variety of mechanisms, including 1) liver cell necrosis 2) reducing first-pass metabolism of a drug through formation of porto-systemic shunting 3) altering production of drug binding proteins 4) inducing renal failure 5) and affecting capacity of hepatic drug metabolizing enzymes leading to abnormal drug volume distribution and altered drug elimination, metabolism and pharmacodynamics (10-12).

In the case of orally administered drugs, cirrhosis may lead to increased bioavailability. These drugs are absorbed from the gastrointestinal tract and transported through the portal vein directly to the liver, via a phenomenon known as the first-pass effect. In this manner they have the opportunity to undergo substantial metabolism in the liver before reaching the systemic circulation. Cirrhosis may lead to formation of portosystemic shunts, which are new blood vessels that divert blood from the abdominal viscera to the heart, bypassing the first passing through liver. As a result, a substantial fraction of the blood, which should normally reach the portal vein, may flow through these shunts, reducing mesenteric blood flow through the liver. For drugs with a high hepatic extraction (low bioavailability in healthy subjects), porto systemic shunting may reduce first-pass metabolism with the consequence of increase in oral bioavailability, which can be associated with a decrease in liver drug clearance (13). Thus, if such orally drugs are administered to cirrhotic patients, their initial dose should be reduced according to the ratio of their hepatic extraction. Furthermore, their maintenance dose has to be adapted irrespective of the route of administration, if possible, according to kinetic studies in cirrhotic patients. In the case of drugs with a low hepatic extraction, bioavailability is not affected by liver disease, but hepatic clearance may be affected. For such drugs, only the maintenance dose has to be reduced, according to the estimated decrease in hepatic drug metabolism. For drugs with an intermediate hepatic extraction, initial oral doses should be chosen in the low range of normal in cirrhotic patients and maintenance doses should be reduced as for high extraction drugs (14). Furthermore, as drug metabolism by liver oxidative CYP enzymes, requires oxygen to function, porto-systemic shunting may reduce the sinusoidal permeability to oxygen and therefore impairs the access of oxygen to hepatocytes, leading to reduction of oxidative function of liver.

Drug metabolism via glucuronidation is less affected than CYP enzymes, possibly because of extra-hepatic glucuronidation (15) or increased production of uridine diphosphate-glucuronyltransferase (UDPGT) in remaining viable hepatocytes (16). In these patients, clearance of some drugs or metabolites that undergo biliary excretion can be reduced as well, requiring dose reduction or avoidance of these drugs. Furthermore, cirrhotic patients often have impaired production of drug-binding proteins such as albumin and $\alpha 1$-acid glycoprotein. Therefore, if a drug is highly protein bound, reduced level of albumin can result in elevated levels of free drug, resulting in increased bioavailability and incidence of toxicity (10).

Hepatorenal syndrome that often occurs in individuals with cirrhosis can complicate drug metabolism particularly with drugs that are mainly eliminated via the kidneys. Patients with this syndrome can display significantly diminished renal drug clearance. Importantly, renal dysfunction may even occur in cirrhotic patients with normal serum creatinine. This phenomenon may happen because of the underproduction of creatinine resulting from diminished muscle mass or decreased rate of hepatic production of creatine, the substrate for creatinine.

Despite renal disease, in which the glomerular filtration rate is the metric used to guide dose adjustment, there is no reliable marker for liver disease severity and hepatic clearance that can be used as a guide for drug dosing. Though various tests like liver function test, indocyanine green clearance, megaxx, Child Pugh score, and MELD score are used for prediction of impaired liver function, they all lack the sensitivity to quantitate the specific ability of the liver to metabolize individual drugs. Thus the usefulness of these measures for dose adjustment purposes is imprecise.

As a general rule, in patients with well-compensated cirrhosis and near-normal liver synthetic function (normal 
Imani F et al.

hepatic synthetic function, normal serum albumin, clotting factors and bilirubin), drug pharmacokinetics are unchanged or modified only to a small extent as compared to patients with decompensated cirrhosis with portal hypertension and significant synthetic dysfunction (14).

\subsection{Use of Analgesics in Patients with Cirrhosis}

Pain management in patients with liver disease poses unique challenges for clinicians. Many of the commonly used analgesics like acetaminophen, NSAIDs and opioids are metabolized through the liver. Adverse events from these pain relievers are frequent, potentially fatal, but often avoidable in patients with chronic liver disease, especially in those with cirrhosis. Prescribing analgesics in these patients often raises the fear of precipitating or worsening renal failure, provoking hepatic encephalopathy, inducing portal hypertension and gastrointestinal bleeding, aggravating hepatic decompensation or causing addiction, especially in patients with a history of alcoholism or other addictive conditions. Because there are no evidence-based guidelines for the use of analgesics in this patient population, the pain management in these patients is challenging.

\subsubsection{Paracetamol (Acetaminophen)}

Paracetamol is commonly used as a first choice analgesic for different nociceptive acute or chronic pain and remains one of the safest available analgesics. However, the use of this drug in patients with hepatic disease is often avoided. This perception arose from awareness of hepatotoxic effect of paracetamol overdose and a lack of understanding its metabolic pathway in patients with liver disease. It is known that the majority of paracetamol is metabolized via the sulfation and glucuronidation pathways into nontoxic products which are then excreted via the urine. However, a small proportion $(<5 \%)$ is oxidized via CYP system, mostly CYP2E1, to a hepatotoxic intermediate, N-acetyl-p-benzoquinone imine (NAPQI), which is readily rendered nontoxic by conjugation to glutathione (17). Pharmacokinetic studies have indicated an increase in the elimination half-life ( $t \frac{1}{2}$ ) of paracetamol in patients with liver cirrhosis compared with healthy controls. Meanwhile, the plasma clearance of the drug was shown to be reduced in these patients (18-20). However, the $t \frac{1}{2}$ of the drug or its plasma clearance has been found to be correlated with prothrombin time as well as the plasma albumin levels.

The theoretical mechanisms of paracetamol-induced hepatotoxicity in chronic cirrhotic patients involves altered metabolism via CYP activity and depleted glutathione stores that cause accumulation of a hepatotoxic intermediate, NAPQI. However, available studies in these patients group have shown that although the half-life of paracetamol may be prolonged, CYP activity is not increased (potentially leading to a lesser degree of NAPQI formation) and glutathione stores are generally sufficient to avoid paracetamol hepatotoxicity (21). As a result, a number of studies claimed that concerns about administration of therapeutic doses of paracetamol in the cirrhotic patients are often inaccurate (21).

Different available studies have evaluated the safety and efficacy of paracetamol administration in cirrhotic patients. It has been indicated that for short-term use or 1-time dosing, up to $4 \mathrm{~g} / \mathrm{d}$ appears to be well tolerated for both healthy and cirrhotic individuals, using alcohol regularly $(22,23)$. This assumption is supported by a double-blind, crossover study of 20 patients with chronic liver disease (including 8 cirrhosis), who tolerated paracetamol at a dosage of $4 \mathrm{~g} / \mathrm{d}$ for 13 days without adverse effects (21). In other survey conducted by Lucena et al. (24), paracetamol was safely used as a dosage of 3 $\mathrm{g} / \mathrm{d}$ even in those with alcoholic cirrhosis. The findings of another case control study evaluating the implication of paracetamol in patients with cirrhosis suggested no association between the occasional use of low dose paracetamol (2-3 g/d) and the acute decompensation of cirrhosis (25).

Nonetheless, another study reported the elevation of aspartate aminotransferase (AST) levels in healthy adults, receiving $4 \mathrm{~g} / \mathrm{d}$ for 14 days (26). Based on this finding, in 2006, the American Liver Foundation (ALF) issued recommendations that people not exceed $3 \mathrm{~g} / \mathrm{d}$ of acetaminophen for any prolonged period of time (27). The ALF noted no issue with short-term use of $4 \mathrm{~g} / \mathrm{d}$. On the basis of data from different studies and with the new FDA guidelines in mind current recommendation for long-term acetaminophen use ( $>14$ days) in cirrhotic patients (not actively drinking alcohol) is for reduced dosing at 2 to $3 \mathrm{~g} / \mathrm{d}(21,28)$.

Because of its proven safety profile (when given in recommended doses) and the lack of sedative effects and absence of nephrotoxicity, paracetamol is the preferred analgesic in patients with liver disease including cirrhosis. However, because paracetamol overdose is known as one of the most common cause of liver failure, it is not surprising that the majority of pain practitioners are not willing to prescribe it to patients with any form of liver disease.

\subsubsection{NSAIDs}

NSAIDs are largely metabolized by CYP enzymes and most are highly protein-bound in plasma (typically > 95\%), usually to albumin. As discussed previously, patients with cirrhosis may have both normal or reduced CYZ activity and the impaired production of drug-binding proteins (29). Thus, altered metabolism and bioavailability of NSAIDs that result in their elevated level in serum can be anticipated in these patients (30).

A major mechanism of NSAIDs-induced adverse effects in patients with advanced stages of cirrhosis is inhibition of prostaglandins (PGs) production. As mentioned before, formation of porto-systemic shunts in cirrhosis leads to a drop in arterial pressure $(31,32)$. In this situation, activation of renin-angiotensin-aldosterone system (RAAS), sympathetic nervous system (SNS) and non- 
osmotic release of antidiuretic hormone (ADH) occur to restore the normal blood homeostasis. These cause more renal sodium and water retention. Persistent renal sodium and water retention plays a major role in a sustained ascites formation. This vascular effect of endogenous vasoconstrictors and the tubular action of antidiuretic hormones are countered by the increased production of vasodilatory renal PGs (33).

Cyclooxygenase (COX) plays a key regulatory role in PGs and thromboxane A2 synthesis (34). The adverse effect of NSAIDs results from its inhibitory action on the activity of both isoforms of COX, including COX-1 and COX-2. COX-1 is constitutively expressed in most tissues and is involved in homeostasis of various physiologic functions, whereas COX-2 is considered an inducible isoform primarily involved in many inflammatory responses $(35,36)$. Thus, by inhabiting both COX-1 and COX-2, non-selective NSAIDs, suppress anti-inflammatory responses (inhibition of COX-2 activity) and also block the synthesis of PGs and thromboxane $\mathrm{A} 2$ in the renal and gastrointestinal (GI) tract (inhibition of COX-1 activity). As a result, their administration causes a profound reduction in renal blood flow, glomerular filtration rate, the cap ability of the kidneys to excrete sodium and water $(34,37,38)$. Furthermore, the reduced platelet synthesis of proaggregatory thromboxane A2 may lead to thrombocytopenia and coagulopathy in cirrhotic patients (39). The significant correlation between the use of NSAIDs and the first bleeding episodes associated with oesophageal or cardiac varices in cirrhotic patients has been found in a case control study (40). The study suggested that the risk of developing this complication in cirrhotic patients using NSAIDs are approximately three times more than cirrhotic patients who do not use these compounds (40). The renal impairment with reduced glomerular filtration rate (GFR), renal blood flow, and sodium and water excretion has been indicated in cirrhotic patients when different NSAIDs including ibuprofen (41, 42), indomethacin (43) aspirin (44) naproxen (45) and sulindac are administered (46).

Selective COX-2 inhibitors are of great interest as an alternative to NSAIDs. Different studies in cirrhotic subjects have shown that renal function is affected by NSAIDs and selective COX-1 inhibitors but not by selective COX-2 inhibitors $(47,48)$. However, available studies on assessment of the safety and efficacy of COX-2 selective inhibitors in patients with cirrhosis are limited. It has been shown that celecoxib (a COX-2 inhibitor drug) administered for 2.5 days in patients with cirrhosis and ascites is not associated with apparent impairment in the renal function or any reduction in mean values of GFR, renal plasma flow, and prostaglandin E2 excretion (45). In a pilot study on nine patients with cirrhosis who received celecoxib for 4 days, no significant changes were observed in the mean values for serum creatinine, GFR, prostaglandin E2 or sodium excretion. However, four of these patients displayed a decrease in GFR greater than 20\% (45).

Regardless of these encouraging findings, experimental evidences have established the constitutive expression of COX-2 in kidney and its implication in maintenance of renal blood flow, mediation of renin release, and regulation of sodium excretion in response to alterations in intravascular volume (49). Therefore, it is difficult to give general advice that COX-2 inhibitors have fewer side effects than non-selective NSAIDs in cirrhotic patients. These findings, together with the lack of studies that evaluate the effect of long-term administration of COX2 inhibitors, lead to the conclusion that administered of these compounds should be with caution or avoided in these patients.

\subsubsection{Opioids}

Liver is the major site of biotransformation for most opioids; thus, the disposition of these drugs may be affected in patients with hepatic insufficiency. Oxidation is the major metabolic pathway of most opioids. The exceptions are morphine and buprenorphine, which primarily undergo glucuronidation, and remifentanil, which is metabolized through ester hydrolysis. As mentioned before, oxidation of drugs may be reduced in patients with hepatic cirrhosis, resulting in decreased drug clearance [for pethidine (meperidine), dextropropoxyphene, pentazocine, tramadol and alfentanil] and/or increased oral bioavailability caused by a reduced first-pass metabolism (for pethidine, dextropropoxyphene, pentazocine and dihydrocodeine). Although prospective epidemiological studies are lacking, opioids commonly contribute to the manifestations of hepatic encephalopathy, and thus they should be avoided in patients with cirrhosis, especially in those with portal hypertension and encephalopathy.

Morphine, an opioid that has been in use since 1827, is metabolized through both CYP pathway and glucuronidation. Different studies have investigated the morphine elimination time in cirrhotic patients. According to most of these studies, the plasma clearance of morphine is significantly reduced, its half-life is prolonged, and its bioavailability is increased in cirrhotic patients compared with individuals with normal liver function (5053). Similarly, hydromorphone has been shown to have increased bioavailability and prolonged half-life in the setting of cirrhosis (54).

Codeine is a pro-drug with no analgesic activity. Its analgesia is due to its conversion to morphine via CYP2d6 in liver. As it is known, capacity of this oxidative enzyme is reduced in patients with compensated liver disease. In this case, the result will be a reduced conversion of codeine to morphine and, in turn, a decrease or lack of analgesia after codeine administration. Furthermore, slow clearance of codeine may cause drug accumulation and depressed ventilation (55). Similarly, hydrocodone and oxycodone are metabolized to hydromorphone and oxymorphone via CYP2d6 and CYP3A4 (56). Thus their ineffective drug metabolism in cirrhotic patients may also result in decreased analgesic efficacy of these compounds. 
Imani F et al.

Meperidine (Pethidine) is a heavily protein bound and predominantly metabolized by hydrolysis to meperidinic acid, which is conjugated and excreted. But it is also metabolized by CYP2B6 and CYP3A4 to normeperidine, a metabolite with serious central nervous system toxicity, neuromuscular irritability and rarely seizures. These adverse effects are more frequent in the presence of renal dysfunction (57). Because of its increased bioavailability (heavily protein bound) and prolonged half-life of its toxic metabolite, meperidine should be avoided in cirrhotic patients.

Although methadone and fentanyl are also heavily protein bound and thus require reduced dosing in patients with cirrhosis, their metabolism does not yield toxic products. As a result these compounds may be better tolerated than meperidine. However, both agents require reduced dosing in patients with hypoalbuminemia. Tramadol is a centrally-acting synthetic analgesic structurally related to codeine and morphine. It is converted by both CYP3A4 and CYP2D6 to O-desmethyltramadol, which is also metabolized by CYP2D6; thus in cirrhotic patients with decreased enzymatic activity, its analgesic properties may not be fully established.

Buprenorphine (Subutex) is a partial mu-opioid receptor agonist with high protein binding affinity. It is metabolized primarily through the liver by CYP3A4 into the active metabolite norbuprenorphine and cleared by glucuronidation and excretion into bile (58). Both buprenorphine and nor buprenorphine are further glucuronidated. Decreased CYP3A4 enzymatic activity in cirrhosis might result in an increase of the bioavailability and decrease of the clearance of buprenorphine. Therefore, dosage should be adjusted.

Generally, if opioids are required for cirrhotic patients, these analgesics should be administrated with a longer interval between doses and possibly lower doses, with individual titration to achieve optimal pain relief without significant adverse effects. Careful follow-up is necessary to check for signs of sedation, opioid-induced constipation and early encephalopathy. Immediate discontinuation of the opioids should be considered if any sign of these complications is appeared. Furthermore, health care practitioners should be aware that using opioids in cirrhotic patients with a history of alcoholism is associated with increased risk of cross-addiction.

\subsection{Physicians' Attitudes Toward Prescribing Anal- gesics}

Even though, cirrhotic patients are more susceptible to the diverse effects of paracetamol, NSAIDs and opioids, guidelines for the use of these drugs in such patients are not available. Data suggest that paracetamol is prescribed rather sparingly for pain relief in cirrhotic patients. In a study by Franz et al. (59) the use of paracetamol was reported in only $6 \%$ of cirrhotic patients. In a web-based questionnaire survey conducted by Rossi et al. (5) the physicians' attitudes regarding the use of paracetamol and NSAIDs in patients with liver disease was evaluated. The results of this work indicated that internists and family physicians were significantly more likely to recommend against the use of paracetamol in patients with compensated cirrhosis compared with gastroenterologists who believed that paracetamol would be safe. In the case of patients with decompensated cirrhosis, $95 \%$ of family physicians and $70 \%$ of internists would not recommend the use of paracetamol compared to just $22 \%$ of gastroenterologists. Even among patients with mild chronic hepatitis without cirrhosis, $15-20 \%$ of general practitioners would not recommend paracetamol. In contrast, none of the gastroenterologists questioned in this survey would avoid paracetamol in that setting. Overall, the recommendation against the use of NSAIDs was significantly less common than recommendations against the use of paracetamol in both compensated and decompensated cirrhosis. Non-gastroenterologists were more likely to recommend NSAIDs compared with gastroenterologists, who in turn, were more likely to recommend the use of paracetamol (5). Given the very real possibility that ibuprofen and other NSA IDs might lead to or worsen GI haemorrhage in patients with underlying gastropathy and coagulopathy (60), the occasional use of paracetamol is in fact preferred over NSAIDs. Unfortunately no study was available evaluating the attitude of physicians toward prescribing opioids.

\section{Conclusions}

In summary, providing safe and effective analgesia to patients with cirrhosis can be a clinical challenge. No evidence-based guidelines exist on the use of analgesics in these patients. Most drugs can be used safely in cirrhosis, including those that are potentially hepatotoxic, but lower doses or reduced dosing frequency is often recommended. In general, for long-term paracetamol use in cirrhotic patients it is recommended to reduce dosing at 2 to $3 \mathrm{~g} / \mathrm{d}$. Although such patients may eliminate paracetamol more slowly than patients without liver disease, repeated administration of the drug does not result in accumulation of hepatotoxic intermediate, NAPQI. Furthermore, there is no evidence of increased CYP enzymes activity or reduced hepatocellular glutathione stores in patients with liver disease.

NSAIDs should be avoided in both compensated and decompensated cirrhotic patients, primarily because of the risk of acute renal failure caused by prostaglandin inhibition. Opioids should be avoided or used sparingly at low and infrequent doses because of the risk of precipitating hepatic encephalopathy. Long-term follow-up for toxicity, adverse effects, and complications is required. Patients with a history of encephalopathy or addiction should not take opioids.

\section{Authors' Contributions}

Study concept and design, critical review of the manu- 
script: Seyed Moayed Alavian and Farnad Imani; Review of Literature and Drafting of the manuscript: Mahsa Motavaf, Saeid Safari.

\section{References}

1. Lim YS, Kim WR. The global impact of hepatic fibrosis and endstage liver disease. Clin Liver Dis. 2008;12(4):733-46.

2. Graudal N, Leth P, Marbjerg L, Galloe AM. Characteristics of cirrhosis undiagnosed during life: a comparative analysis of 73 undiagnosed cases and 149 diagnosed cases of cirrhosis, detected in 4929 consecutive autopsies. J Intern Med.1991;230(2):165-71.

3. Alavian SM, Fallahian F, Lankarani KB. The changing epidemiology of viral hepatitis B in Iran. J Gastrointestin Liver Dis. 2007;16(4):403-6.

4. Keyvani H, Bokharaei-Salim F, Monavari SH, Esghaei M, Nassiri Toosi M, Fakhim S, et al. Occult hepatitis $\mathrm{C}$ virus infection in candidates for liver transplant with cryptogenic cirrhosis. Hepat Mon. 2013;13(8).

5. Rossi S, Assis DN, Awsare M, Brunner M, Skole K, Rai J, et al. Use of over-the-counter analgesics in patients with chronic liver disease: physicians' recommendations. Drug Saf. 2008;31(3):261-70.

6. Imani F, Safari S. "Pain Relief is an Essential Human Right", We Should be Concerned about It. Anesth Pain Med. 2011;1(2):55-7.

7. Elbekai RH, Korashy HM, El-Kadi AO. The effect of liver cirrhosis on the regulation and expression of drug metabolizing enzymes. Curr Drug Metab. 2004;5(2):157-67.

8. Bohan A, Boyer JL. Mechanisms of hepatic transport of drugs: implications for cholestatic drug reactions. Semin Liver Dis. 2002;22(2):123-36.

9. Rahimzadeh P, Safari S, Faiz SH, Alavian SM. Anesthesia for patients with liver disease. Hepat Mon. 2014;14(7).

10. Verbeeck RK. Pharmacokinetics and dosage adjustment in patients with hepatic dysfunction. Eur J Clin Pharmacol. 2008;64(12):1147-61.

11. Safari S, Motavaf M, Seyed Siamdoust SA, Alavian SM. Hepatotoxicity of Halogenated Inhalational Anesthetics. Iran Red Crescent Med J. 2014;16(9).

12. Mohseni M, Safari S, Alavian SM. Volatile anesthetics in ischemic liver injury: enemy or friend? Hepat Mon. 2014;14(6).

13. Blaschke TF, Rubin PC. Hepatic first-pass metabolism in liver disease. Clin Pharmacokinet.1979;4(6):423-32.

14. Delco F, Tchambaz L, Schlienger R, Drewe J, Krahenbuhl S. Dose adjustment in patients with liver disease. Drug Saf. 2005;28(6):529-45.

15. Hoyumpa AM, Schenker S. Is glucuronidation truly preserved in patients with liver disease? Hepatology. 1991;13(4):786-95.

16. Debinski HS, Lee CS, Danks JA, Mackenzie PI, Desmond PV. Localization of uridine 5 '-diphosphate-glucuronosyltransferase in human liver injury. Gastroenterology. 1995;108(5):1464-9.

17. Graham GG, Scott KF, Day RO. Tolerability of paracetamol. Drug Saf. 2005;28(3):227-40.

18. Arnman R, Olsson R. Elimination of paracetamol in chronic liver disease. Acta Hepatogastroenterol (Stuttg). 1978;25(4):283-6.

19. Andreasen PB, Hutters L. Paracetamol (acetaminophen) clearance in patients with cirrhosis of the liver. Acta Med Scand Suppl. 1979;624:99-105.

20. Alimian M, Pournajafian A, Kholdebarin A, Ghodraty M, Rokhtabnak F, Yazdkhasti P. Analgesic effects of paracetamol and morphine after elective laparotomy surgeries. Anesth Pain Med. 2014;4(2).

21. Benson GD, Koff RS, Tolman KG. The therapeutic use of acetaminophen in patients with liver disease. Am JTher. 2005;12(2):133-41.

22. Kuffner EK, Green JL, Bogdan GM, Knox PC, Palmer RB, Heard K, et al. The effect of acetaminophen (four grams a day for three consecutive days) on hepatic tests in alcoholic patients--a multicenter randomized study. BMC Med. 2007;5:13.

23. Heard K, Green JL, Bailey JE, Bogdan GM, Dart RC. A randomized trial to determine the change in alanine aminotransferase during 10 days of paracetamol (acetaminophen) administration in subjects who consume moderate amounts of alcohol. Aliment Pharmacol Ther. 2007;26(2):283-90.
24. Lucena MI, Andrade RJ, Tognoni G, Hidalgo R, Sanchez de la Cuesta F, Spanish Collaborative Study Group on Therapeutic Management of Liver D. Drug use for non-hepatic associated conditions in patients with liver cirrhosis. Eur J Clin Pharmacol. 2003;59(1):71-6.

25. Khalid SK, Lane J, Navarro V, Garcia-Tsao G. Use of over-the-counter analgesics is not associated with acute decompensation in patients with cirrhosis. Clin Gastroenterol Hepatol. 2009;7(9):994-9.

26. Watkins PB, Kaplowitz N, Slattery JT, Colonese CR, Colucci SV, Stewart PW, et al. Aminotransferase elevations in healthy adults receiving 4 grams of acetaminophen daily: a randomized controlled trial. JAMA. 2006;296(1):87-93.

27. American Liver Foundation. The American Liver Foundation Issues Warning on Dangers of Excess Acetaminophen. American Liver Foundation. 2006. Available from: http://www.liverfoundation.org/ downloads/alf_download_39.pdf.

28. Chandok N, Watt KD. Pain management in the cirrhotic patient: the clinical challenge. Mayo Clin Proc. 2010;85(5):451-8.

29. Schoene B, Fleischmann RA, Remmer H, von Oldershausen HF Determination of drug metabolizing enzymes in needle biopsies of human liver. EurJ Clin Pharmacol.1972;4(2):65-73.

30. Williams RL, Upton RA, Cello JP, Jones RM, Blitstein M, Kelly J, et al. Naproxen disposition in patients with alcoholic cirrhosis. Eur JClin Pharmacol.1984;27(3):291-6.

31. Hartleb M, Gutkowski K. Kidneys in chronic liver diseases. World J Gastroenterol. 2012;18(24):3035-49.

32. Wong F, Massie D, Hsu P, Dudley F. Indomethacin-induced renal dysfunction in patients with well-compensated cirrhosis. Gastroenterology. 1993;104(3):869-76.

33. Laffi G, La Villa G, Pinzani M, Marra F, Gentilini P.Arachidonic acid derivatives and renal function in liver cirrhosis. Semin Nephrol. 1997;17(6):530-48.

34. Arroyo V, Gines P, Rimola A, Gaya J. Renal function abnormalities, prostaglandins, and effects of nonsteroidal anti-inflammatory drugs in cirrhosis with ascites. An overview with emphasis on pathogenesis. Am J Med.1986;81(2B):104-22.

35. O'Neill GP, Ford-Hutchinson AW. Expression of mRNA for cyclooxygenase-1 and cyclooxygenase-2 in human tissues. FEBS Lett. 1993;330(2):156-60.

36. Morita I. Distinct functions of COX-1 and COX-2. Prostaglandins Other Lipid Mediat. 2002;68-69:165-75.

37. Perez-Ayuso RM, Arroyo V, Camps J, Rimola A, Gaya J, Costa J, et al Evidence that renal prostaglandins are involved in renal water metabolism in cirrhosis. Kidney Int. 1984;26(1):72-80.

38. Warner TD, Giuliano F, Vojnovic I, Bukasa A, Mitchell JA, Vane JR. Nonsteroid drug selectivities for cyclo-oxygenase-1 rather than cyclo-oxygenase- 2 are associated with human gastrointestinal toxicity: a full in vitro analysis. Proc Natl Acad Sci U S A. 1999;96(13):7563-8.

39. Peck-Radosavljevic M. Review article: coagulation disorders in chronic liver disease. Aliment Pharmacol Ther. 2007;26 Suppl 1:21-8.

40. De Ledinghen V, Heresbach D, Fourdan O, Bernard P, Liebaert-Bories MP, Nousbaum JB, et al. Anti-inflammatory drugs and variceal bleeding: a case-control study. Gut.1999;44(2):270-3.

41. Ackerman Z, Cominelli F, Reynolds TB. Effect of misoprostol on ibuprofen-induced renal dysfunction in patients with decompensated cirrhosis: results of a double-blind placebo-controlled parallel group study. Am J Gastroenterol. 2002;97(8):2033-9.

42. Laffi G, Daskalopoulos G, Kronborg I, Hsueh W, Gentilini P, Zipser RD. Effects of sulindac and ibuprofen in patients with cirrhosis and ascites. An explanation for the renal-sparing effect of sulindac. Gastroenterology. 1986;90(1):182-7.

43. Zipser RD, Hoefs JC, Speckart PF, Zia PK, Horton R. Prostaglandins: modulators of renal function and pressor resistance in chronic liver disease. J Clin Endocrinol Metab.1979;48(6):895-900.

44. Arroyo V, Planas R, Gaya J, Deulofeu R, Rimola A, Perez-Ayuso RM, et al. Sympathetic nervous activity, renin-angiotensin system and renal excretion of prostaglandin E2 in cirrhosis. Relationship to functional renal failure and sodium and water excretion. Eur J Clin Invest. 1983;13(3):271-8.

45. Claria J, Kent JD, Lopez-Parra M, Escolar G, Ruiz-Del-Arbol L, Gines $P$, et al. Effects of celecoxib and naproxen on renal function in 
nonazotemic patients with cirrhosis and ascites. Hepatology. 2005;41(3):579-87.

46. Brater DC, Anderson SA, Brown-Cartwright D. Reversible acute decrease in renal function by NSAIDs in cirrhosis. Am J Med Sci. 1987;294(3):168-74.

47. Bosch-Marce M, Claria J, Titos E, Masferrer JL, Altuna R, Poo JL, et al. Selective inhibition of cyclooxygenase 2 spares renal function and prostaglandin synthesis in cirrhotic rats with ascites. Gastroenterology. 1999;:116(5):1167-75.

48. Lopez-Parra M, Claria J, Planaguma A, Titos E, Masferrer JL, Woerner BM, et al. Cyclooxygenase-1 derived prostaglandins are involved in the maintenance of renal function in rats with cirrhosis and ascites. BrJ Pharmacol. 2002;135(4):891-900.

49. Harris RC. COX-2 and the kidney.J Cardiovasc Pharmacol. 2006;47 Suppl 1:S37-42.

50. Hasselstrom J, Eriksson S, Persson A, Rane A, Svensson JO, Sawe $J$. The metabolism and bioavailability of morphine in patients with severe liver cirrhosis. BrJ Clin Pharmacol. 1990;29(3):289-97.

51. Hajiesmaeili MR, Safari S. Pain management in the intensive care unit: do we need special protocols? Anesth Pain Med. 2012;1(4):237-8

52. Eydi M, Golzari SE, Aghamohammadi D, Kolahdouzan K, Safari S, Ostadi Z. Postoperative Management of Shivering: A Comparison of Pethidine vs. Ketamine. Anesth Pain Med. 2014;4(2).
53. Imani F, Faiz HR, Sedaghat M, Hajiashrafi M. Effects of adding ketamine to fentanyl plus acetaminophen on postoperative pain by patient controlled analgesia in abdominal surgery. Anesth Pain Med. 2014;4(1).

54. Durnin C, Hind ID, Ghani SP, Yates DB, Molz KH. Pharmacokinetics of oral immediate-release hydromorphone (Dilaudid IR) in subjects with moderate hepatic impairment. Proc West Pharmacol Soc. 2001;44:83-4.

55. Tallgren M, Olkkola KT, Seppala T, Hockerstedt K, Lindgren L. Pharmacokinetics and ventilatory effects of oxycodone before and after liver transplantation. Clin Pharmacol Ther. 1997; 61(6):655-61.

56. Moradi M, Esmaeili S, Shoar S, Safari S. Use of oxycodone in pain management. Anesth Pain Med. 2012;1(4):262-4.

57. Smith HS. Opioid metabolism. Mayo Clin Proc. 2009;84(7):613-24.

58. Iribarne C, Picart D, Dreano Y, Bail JP, Berthou F. Involvement of cytochrome P450 3A4 in N-dealkylation of buprenorphine in human liver microsomes. Life Sci. 1997;60(22):1953-64.

59. Franz CC, Egger S, Born C, Ratz Bravo AE, Krahenbuhl S. Potential drug-drug interactions and adverse drug reactions in patients with liver cirrhosis. Eur J Clin Pharmacol. 2012;68(2):179-88.

60. Lee YC, Chang CH, Lin JW, Chen HC, Lin MS, Lai MS. Non-steroidal anti-inflammatory drugs use and risk of upper gastrointestinal adverse events in cirrhotic patients. Liver Int. 2012;32(5):859-66. 